\title{
The characteristic expression pattern of $B M I-1$ and SALL4 genes in placenta tissue and cord blood
}

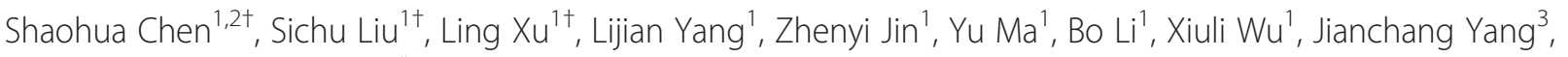
Yupo $\mathrm{Ma}^{3}$ and Yangqiu Li, ${ }^{1,2^{*}}$

\begin{abstract}
Introduction: SALL4 and BMI-1 are important factors in hematopoiesis. Placental tissue (PT) and umbilical cord blood (CB) are rich in hematopoietic stem/progenitor cells (HSCS/HPCS), but their SALL4 and BMI-1 expression levels remain unknown.

Methods: Real-time PCR was used to determine the expression level of these genes in PT and CB from ten cases, and ten healthy donors were used as controls.

Results: A significantly higher BMI-1 and SALL4 gene expression level was found in PT (median: 17.548 and 34.362, respectively) than in cord blood mononuclear cells (CBMCs) (median: 2.071 and 11.300 , respectively) $(P=0.0001$ and $P=0.007)$ and healthy peripheral blood mononuclear cells (PBMCs) (median: 0.259 and 0.384 , respectively) $(P=0.001$ and $P<0.0001)$, and their expression level was lower in PBMCs than in CBMCs $(P=0.029$ and $P=0.002)$. A positive correlation between the BMI-1 and SALL4 genes was found in the PT and CB groups, while there was no significant correlation between these genes in the healthy group. There was also no significant correlation between the expression level of each gene in PT and CB.

Conclusions: These results describe the characteristic features of the BMI-1 and SALL4 gene expression pattern in placental tissue and cord blood. Placental tissue with higher expression level of both genes may be considered as a potential resource for SALL4-related HPC expansion.
\end{abstract}

Keywords: BMI-1 gene, Cord blood, Placenta, Real-time PCR, SALL4 gene

\section{Introduction}

Umbilical cord blood (CB) is a valuable alternative hematopoietic stem cells (HSC) source for transplantations for patients who lack a suitable sibling donor [1-3]. It has been demonstrated previously that $\mathrm{CB}$-derived progenitors can express Oct3/4, SRY-related HMG-box 2 (Sox2), Nanog and reduced expression-1 (Rex1), which are pluripotent/multilineage markers and could potentially differentiate into multiple lineages $[4,5]$. However, different gene expression pattern may determine the use of human CB-derived HSCs/hematopoietic progenitor cells (HPCs) as functional tissues or cells [6].

\footnotetext{
* Correspondence: yangqiuli@hotmail.com

${ }^{\dagger}$ Equal contributors

'Key Laboratory for Regenerative Medicine of Ministry of Education, Jinan University, Guangzhou 510632, China

${ }^{2}$ Institute of Hematology, Jinan University, Guangzhou 510632, China

Full list of author information is available at the end of the article
}

Sal-like protein 4 (SALL4) and B cell-specific MLV integration site 1 (BMI-1) are important factors in hematopoiesis and are expressed in hematopoietic stem/ progenitor cells (HSCs/HPCs) and myeloid leukemia cells [7]. Placental tissue (PT) and CB comprise rich HSCs/HPCs; however, little is known about the difference in the expression level of SALL4 and BMI-1 in PT and $\mathrm{CB}$.

SALL4, a newly identified zinc-finger transcription factor that is a member of the $S A L L$ gene family, was originally cloned based on its sequence homology to Drosophila spalt $(\mathrm{sal})[7,8]$. This protein plays important roles in maintaining embryonic stem cells (ESC) pluripotency and $\mathrm{HSC} / \mathrm{HPC}$ self-renewal properties, and it has been recently proposed for use in $\mathrm{CB}$ expansion [9]. Recently, it was demonstrated that BMI-1 is a direct SALL4 target gene. BMI-1 is a member of the polycomb group of proteins, and it was initially identified in

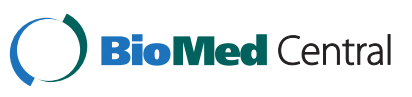


Drosophila as a repressor of homeotic genes $[7,10]$. This protein is highly expressed in purified HSCs, its expression declines with differentiation $[7,11]$, and it plays an essential role in regulating adult, self-renewing HSCs/HPCs [7,11-13]. The induction of SALL4 expression is associated with increased levels of histone H3-K4 and H3-K79 methylation in the $B M I-1$ promoter, indicating a novel connection between SALL4 and the polycomb group proteins [7].

In this study, we sought to characterize the expression pattern of the BMI-1 and SALL4 genes in PT and CB.

\section{Materials and methods Samples}

Ten placental tissue samples were obtained from fullterm deliveries, and umbilical cord blood was obtained at the same time from full-term healthy babies with the mothers' consent. All human tissue and cell samples were obtained with consent from the human subjects. Peripheral blood samples from ten healthy donors with informed consent were used as control. All of the procedures were conducted according to the guidelines of the Medical Ethics Committees of the Health Bureau of the Guangdong Province of China, and ethical approval was obtained from the Ethics Committee of Medical School of Jinan University for this study.

\section{Quantitative real-time reverse transcription-polymerase chain reaction ( $\mathrm{qRT}-\mathrm{PCR}$ )}

Mononuclear cells were isolated from cord blood samples (CBMCs) and healthy peripheral blood (PBMCs) by Ficoll-Hypaque gradient centrifugation. The placental tissue (approximately $100 \mathrm{mg}$ ) was obtained by curettage of the central portion of the placenta (decidua in majority). RNA isolation and cDNA synthesis were performed according to the manufacturer's protocol [14].
The expression level of BMI-1, SALL4, and the $\beta 2$ microglobulin $(\beta 2-M G)$ reference gene was determined by SYBR Green I real-time PCR as previously described [15].

\section{Statistical analyses}

Differences in mRNA expression between two groups were analyzed using the Mann-Whitney test. Data are presented as the median. Spearman's rank correlation analysis was used to analyze the SALL4 and BMI-1 mRNA levels in different samples. Differences were considered statistically significant with a $P<0.05$.

\section{Results and Discussion}

Due to its wide availability, low cost and lack of ethical concerns, CB provides an attractive source of stem cells for investigational and therapeutic uses [1-3]. Recently, in the promising field of regenerative medicine, human perinatal stem cells that could be isolated from normally discarded human placentae are of great interest as potential stem cells with clinical applications. Perinatal stem cells are an ideal cell source in terms of availability, the fewer number of ethical concerns, less DNA damage, and so on [16,17]. The biological characteristics of CBderived HSCs/HPCs were characterized in numerous studies [4,6]. SALL4 may act as a critical regulator of the fate of hematopoietic cells. In normal bone marrow, SALL4 is selectively expressed in primitive hematopoietic precursors and is rapidly downregulated following differentiation. Therefore, the SALL4 gene expression level should be positively correlated with stem cells [7]. However, little is known about the difference in the expression level of SALL4 in CB; thus, it may be interesting to determine the expression characteristics of this gene in PT collected from the same case as the $\mathrm{CB}$ to compare expression pattern differences. In this study, we found that the SALL4 expression level was higher in PT
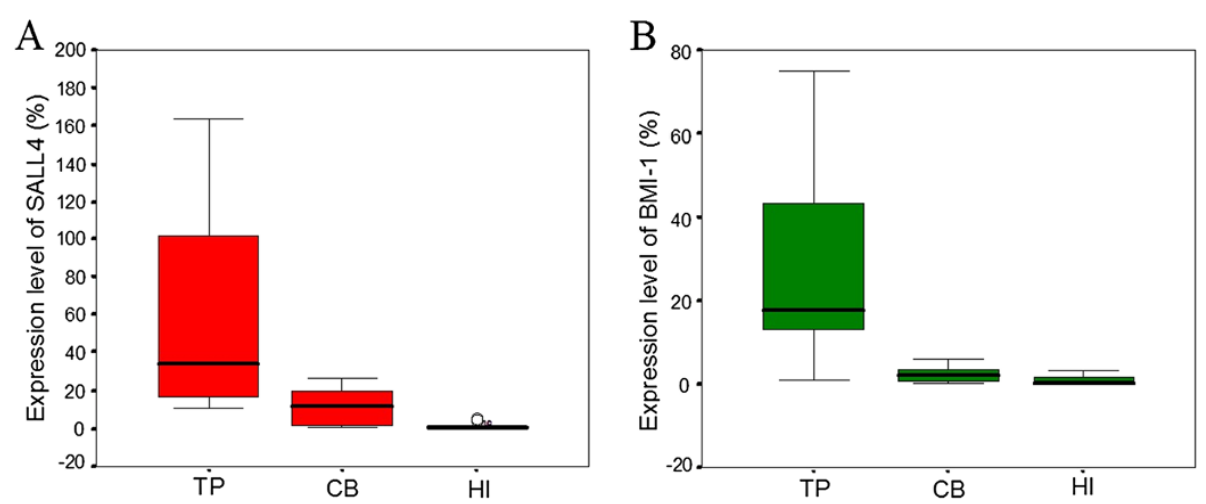

Figure 1 Expression level of SALL4 and BMI-1. Expression level of the SALL4 (A) and BMI-1 (B) genes in placental tissue (PT), cord blood and peripheral blood mononuclear cells (CBMCs and PBMCs, respectively) from healthy individuals (HI). A significant difference was found among the three groups. 

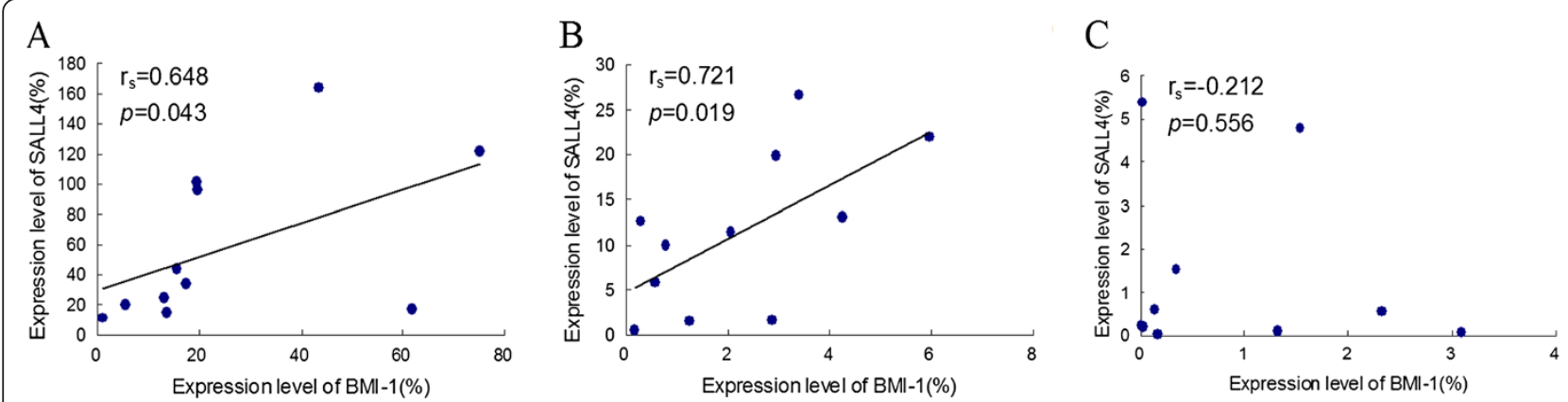

Figure 2 Correlation of expression level of SALL-4 and BMI-1 in different samples. Correlation analysis of the relative expression level of the BMI-1 and SALL4 genes in placental tissue (PT) (A), cord blood mononuclear cells (CBMCs) (B) and peripheral blood mononuclear cells (PBMCs) (C).

(median: 34.362) than that in CBMCs (median: 11.300; $P=0.007$ ) and PBMCs (median: 0.384; $P<0.0001$ ), and a lower SALL4 expression level was found in PBMCs than CBMCs (Figure 1A; $P=0.002$ ). It has been reported that SALL4-expanded HSCs/HPCs retain multilineage repopulation and long-term engraftment abilities, which are clinically significant [18]. Thus, SALL4 could be used to stimulate the large scale ex vivo expansion of HSCs/ HPCs, and it is particularly interesting whether SALL4rich placental tissue is able to be used as a resource for HSC/HPC expansion.

Because BMI-1 is a direct SALL4 target gene [7], $B M I-1$ expression may be correlated with that of SALL4. Our results also demonstrated that the BMI-1 gene expression level was significantly higher in PT (median: 17.548) than that in CBMCs (median: $2.071 ; P=0.0001$ ) and PBMCs (median: 0.259; $P=0.001$ ), and its expression level was lower in PBMCs than that in CBMCs (Figure 1B; $P=0.029$ ). A positive correlation between the BMI-1 and SALL4 genes was found in the PT and $\mathrm{CB}$ groups $(\mathrm{rs}=0.648, P=0.043$ and $\mathrm{rs}=0.721$, $P=0.019$, respectively), while there was no significant correlation between these genes in the healthy group (rs $=-0.212, P=0.556$ ) (Figure 2). This result further supports the finding of a relationship between the expression pattern of BMI-1 and SALL4 in HSC/HPC-rich
$\mathrm{CB}$ and placental tissue, while PBMCs contain only limited BMI-1 and SALL4 expression, which may reflect their expression in different levels of HPCs and myeloid cells [7]. Moreover, this result may indicate that the PT niche contains hematopoietic transcription factors that are able to enhance HPC expansion. It is thought that the expression level of genes in PT and CB derived from the same case may demonstrate a positive correlation; however, interestingly, the expression levels of BMI-1 and SALL4 were not significantly correlated between the PT and $C B$ (Figure 3). This result may be related to a difference in the HSC/HPC numbers in CB or may imply that placental tissue-derived HSCs/HPCs have a different SALL4 and $B M I-1$ expression pattern. Further analysis is needed to compare the expression levels of these genes and the percentages of HSCs/HPCs in CB and PT.

\section{Conclusions}

We determined the expression characteristics of BMI-1 and SALL4 in placental tissue and cord blood. The results of this study may contribute to a better understanding of the expression characteristics and correlation of SALL4 and BMI-1 in placenta tissue. Placental tissue with higher expression level of both genes may be considered as a potential resource for SALL4-related HPC expansion.
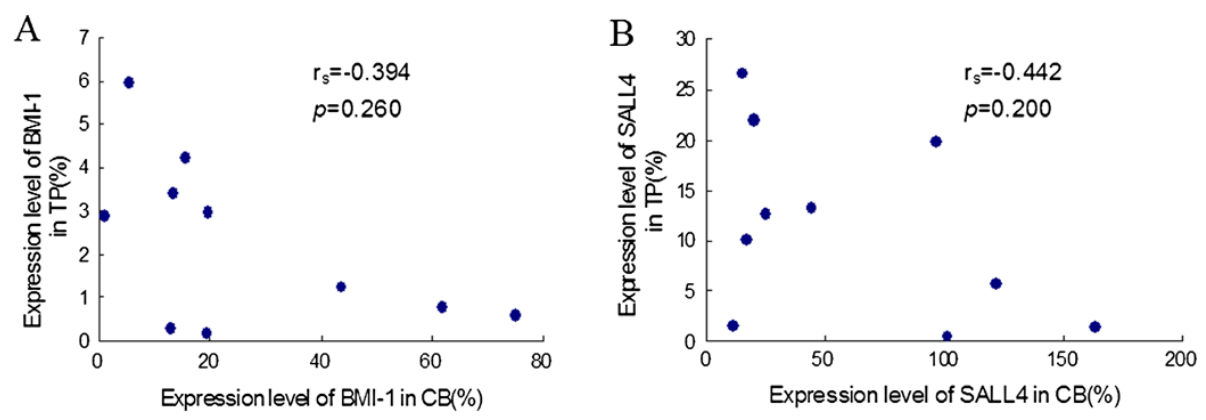

Figure 3 Correlation of expression level of BMI-1 and SALL4 between PT and CB. Correlation analysis of the relative expression level of the BMI-1 (A) and SALL4 (B) genes in placental tissue (PT) and cord blood mononuclear cells (CBMCS). 


\section{Abbreviations}

BMI-1: B cell-specific MLV integration site 1 gene; CB: umbilical cord blood; CBMCs: cord blood mononuclear cells; ESC: embryonic stem cells; HPCs: hematopoietic progenitor cells; HSCs: hematopoietic stem cells; PBMCs: healthy peripheral blood mononuclear cells; PT: placental tissue; qRT-PCR: quantitative real-time reverse transcription-polymerase chain reaction; Rex1: reduced expression 1; SALL4: Sal-like protein 4 gene; Sox2: SRY-related HMG-box 2; $\beta 2-M G: ~ \beta 2$ microglobulin.

\section{Competing interests}

The authors declare that they have no competing interests.

\section{Authors' contributions}

$Y L, Y M$ and JY contributed to the concept development and study design. SC, SL, LX, ZJ, and YM performed real-time PCR experiments. LY, BL and XW were responsible for the collection of clinical samples. YL, SC, SL and LX coordinated the study and helped in drafting the manuscript. All authors read and approved the final manuscript.

\section{Acknowledgements}

This study was supported by grants from the National Natural Science Foundation of China (no. 81270604), the Collaborated grant for HK-Macao-TW of Ministry of Science and Technology (2012DFH30060), the Fundamental Research Funds for Central Universities (no. 21612116), and, in part, an NIH grant to YM (no. R01HL087948).

\section{Author details}

${ }^{1}$ Key Laboratory for Regenerative Medicine of Ministry of Education, Jinan University, Guangzhou 510632, China. ${ }^{2}$ Institute of Hematology, Jinan University, Guangzhou 510632, China. ${ }^{3}$ Department of Pathology, BST-9, School of Medicine, The State University of New York at Stony Brook, Stony Brook, NY 11794, USA.

Received: 16 March 2013 Revised: 12 April 2013

Accepted: 26 April 2013 Published: 30 April 2013

\section{References}

1. Brunstein CG, Baker KS, Wagner JE: Umbilical cord blood transplantation for myeloid malignancies. Curr Opin Hematol 2007, 14:162-169.

2. LV M, Huang XJ: Allogeneic hematopoietic stem cell transplantation in China: where we are and where to go. J Hematol Oncol 2012, 5:10.

3. Zhou F, Ge L, Yu Z, Fang Y, Kong F: Clinical observations on intensive immunosuppressive therapy combined with umbilical cord blood support for the treatment of severe aplastic anemia. 1 Hematol Oncol 2011, 4:27.

4. Divya MS, Roshin GE, Divya TS, Rasheed VA, Santhoshkumar TR, Elizabeth KE, James J, Pillai RM: Umbilical cord blood-derived mesenchymal stem cells consist of a unique population of progenitors co-expressing mesenchymal stem cell and neuronal markers capable of instantaneous neuronal differentiation. Stem Cell Res Ther 2012, 3:57.

5. Lim JY, Jeong $\mathrm{CH}$, Jun JA, Kim SM, Ryu CH, Hou Y, Oh W, Chang JW, Jeun SS: Therapeutic effects of human umbilical cord blood-derived mesenchymal stem cells after intrathecal administration by lumbar puncture in a rat model of cerebral ischemia. Stem Cell Res Ther 2011, 2:38.

6. Berger MJ, Minnerath SR, Adams SD, Tigges BM, Sprague SL, McKenna DH $\mathrm{Jr}$ : Gene expression changes with differentiation of cord blood stem cells to respiratory epithelial cells: a preliminary observation. Stem Cell Res Ther 2011, 2:19.

7. Yang JC, Chai L, Liu F, Fink LM, Lin P, Silberstein LE, Amin HM, Ward DC, Ma YP: Bmi-1 is a target gene for SALL4 in hematopoietic and leukemic cells. Proc Natl Acad Sci USA 2007, 104:10494-10499.

8. Al-Baradie R, Yamada K, St Hilaire C, Chan WM, Andrews C, Mclntosh N, Nakano M, Martonyi EJ, Raymond WR, Okumura S, Okihiro MM, Engle EC: Duane radial ray syndrome (Okihiro syndrome) maps to $20 \mathrm{q} 13$ and results from mutations in SALL4, a new member of the SAL family. Am J Hum Genet 2002, 71:1195-1199.

9. Schuster JA, Stupnikov MR, Ma G, Liao WB, Lai R, Ma YP, Aguila JR: Expansion of hematopoietic stem cells for transplantation: current perspectives. Exp Hematol Oncol 2012, 1:12.
10. Franke A, DeCamillis M, Zink D, Cheng N, Brock HW, Paro R: Polycomb and polyhomeotic are constituents of a multimeric protein complex in chromatin of Drosophila melanogaster. EMBO J 1992, 11:2941-2950.

11. Park IK, Qian D, Kiel M, Becker MW, Pihalja M, Weissman IL, Morrison SJ, Clarke MF: Bmi-1 is required for maintenance of adult self-renewing haematopoietic stem cells. Nature 2003, 423:302-305.

12. Raaphorst FM: Self-renewal of hematopoietic and leukemic stem cells: a central role for the Polycomb-group gene Bmi-1. Trends Immunol 2003, 24:522-524.

13. Iwama A, Oguro H, Negishi M, Kato Y, Morita Y, Tsukui H, Ema H, Kamijo T, Katoh-Fukui $Y$, Koseki $H$, van Lohuizen M, Nakauchi H: Enhanced selfrenewal of hematopoietic stem cells mediated by the polycomb gene product Bmi-1. Immunity 2004, 21:843-851.

14. Lu X, Chen SH, Yang L, Zha X, Yang X, Li Y: The feature of expression pattern of CD3 chain genes in fetal-maternal interface. Hematology 2011, 16:185-189

15. Shen Q, Liu S, Hu J, Chen S, Yang L, Li B, Wu X, Ma Y, Yang J, Ma Y, Li Y. The differential expression pattern of the BMI-1, SALL4 and ABCA3 genes in myeloid leukemia. Cancer Cell Int 2012, 12:42

16. Miki T: Amnion-derived stem cells: in quest of clinical applications. Stem Cell Res Ther 2011, 2:25.

17. Siqueira RC: Stem cell therapy for retinal diseases: update. Stem Cell Res Ther 2011, 2:50.

18. Gao C, Kong NR, Li A, Tatetu H, Ueno S, Yang Y, He J, Yang J, Ma Y, Kao GS, Tenen DG, Chai L: SALL4 is a key transcription regulator in normal human hematopoiesis. Transfusion. In press.

doi:10.1186/scrt199

Cite this article as: Chen et al:: The characteristic expression pattern of BMI-1 and SALL4 genes in placenta tissue and cord blood. Stem Cell Research \& Therapy 2013 4:49.

\section{Submit your next manuscript to BioMed Central and take full advantage of:}

- Convenient online submission

- Thorough peer review

- No space constraints or color figure charges

- Immediate publication on acceptance

- Inclusion in PubMed, CAS, Scopus and Google Scholar

- Research which is freely available for redistribution 